\section{Endovascular Procedures in Elderly Patients}

When one reads "Age-Related Complications following Endovascular Treatment of Unruptured Intracranial Aneurysms," one should expect an article that focuses on unexpected events, strictly associated with the aging process during an endovascular procedure. ${ }^{1}$

Elderly patients who undergo invasive procedures are prone to complications, mostly related to atherosclerotic hardening and plugging of the arteries, arrhythmias, autoregulation disturbances, cardiovascular insufficiency, and endocrine and renal failure, among others related to muscle weakness and degenerative osseous changes. Although there are some controversies about this subject, ${ }^{2}$ we agree with the predisposition of elderly people to complications in surgical and endovascular procedures, ${ }^{2,3}$ but we do not think the design and results of Khosla et $\mathrm{al}^{1}$ are strong enough support this predisposition.

Of the 110 complications, 41 (37\%) were major. Among them, $26(6.6 \%$ of all procedures) did not result in neurologic deficits at discharge, and these included temporary neurologic deficits $(n=13$, $3.3 \%)$, subarachnoid hemorrhage $(n=8,2.0 \%)$, acute anemia requiring transfusion $(n=3,0.8 \%)$, seizures $(n=1,0.25 \%)$, and 1 patient with an occlusion of the common femoral artery requiring thrombectomy $(0.25 \%)$.

According the previous data, there was a significant statistical difference between nonelderly and elderly patients (7.4\% versus $17 \%)$, but this was general and nonspecific information. The authors gave only details about the ones that might be aggravated by the aging process.

We found some inconsistencies in this analysis. For example, temporary neurologic deficits outnumbered other complications, but how can we explain them? During endovascular procedures, distal embolism or vasospasm is the most common cause of transient deficits; therefore were the intravascular thrombi or emboli, which occurred in 30 patients $(7.6 \%)$, really asymptomatic? Could the authors provide any other plausible reason for these deficits, or could they just be secondary to the known difficult arterial navigation in elderly patients? However, even if this was the actual reason, why use "compromised vascular reserve" as an explanation? Then, what criteria did the authors use to differentiate these cases from the ones with neurologic deficits after the procedure: 13 patients $(3.3 \%)$ with ischemic infarctions and 2 patients $(0.5 \%)$ with fatal infarctions?

On the other hand, why were subarachnoid hemorrhages, acute anemia, or seizures increased in the elderly group, or were they just unwanted but "expected" results of the endovascular procedure? We cannot see, either, the relationship between age and increased risk of this therapy in this group.

Regardless of these opinions, the high proficiency of Khosla et $\mathrm{al}^{1}$ in dealing with this complex medical problem is clear; therefore, we encourage them to continue the research into this subject with a prospective and well-designed study.

\section{References}

1. Khosla A, Brinjikji W, Cloft H, et al. Age-related complications following endovascular treatment of unruptured intracranial aneurysms. AJNR Am J Neuroradiol 2012;33:953-57

2. Ryttlefors M, Enblad P, Kerr RS, et al. International subarachnoid aneurysm trial of neurosurgical clipping versus endovascular coiling: subgroup analysis of 278 elderly patients. Stroke 2008;39:2720-26

3. Johansson M, Norback O, Gal G, et al. Clinical outcome after endovascular coil embolization in elderly patients with subarachnoid hemorrhage. Neuroradiology 2004;46:385-91

R.A.P. Falero

O.L. Piloto

Department of Neurosurgery Hospital Hermanos Ameijeiras La Habana, Cuba

http://dx.doi.org/10.3174/ajnr.A3360 